\title{
Design of an Antioxidant Nitrone Scaffold Based on Plant Pigments
}

Amanda Capistrano Pinheiro, ${ }^{\text {a }}$ Rodrigo Boni Fazzi, ${ }^{a}$ Larissa Cerrato Esteves, ${ }^{\text {a }}$ Caroline Oliveira Machado, ${ }^{\text {a }}$ Felipe Augusto Dörr, ${ }^{\mathrm{b}}$ Ernani Pinto, ${ }^{\mathrm{b}}$ Yocefu Hattori, ${ }^{\mathrm{c}}$ Jacinto Sa, ${ }^{\mathrm{c}, \mathrm{d}}$ Ana Maria da Costa Ferreira $^{\mathrm{a}}$ and Erick Leite Bastos*a

a Departamento de Química Fundamental, Instituto de Química, Universidade de São Paulo, 05508-000 São Paulo, SP, Brazil.

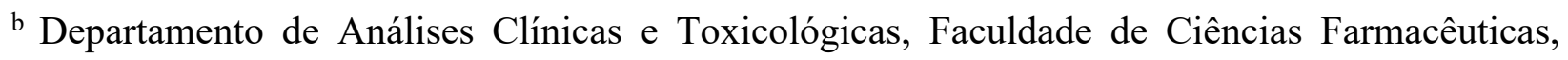
Universidade de São Paulo, 05508-000 São Paulo, SP, Brazil

c Physical Chemistry Division, Department of Chemistry, Ångström Laboratory, Uppsala University, 75120 Uppsala, Sweden

${ }^{\mathrm{d}}$ Institute of Physical Chemistry, Polish Academy of Sciences, 01-224 Warsaw, Poland

* Corresponding author: elbastos@iq.usp.br 


\section{ABSTRACT}

Nitrones derived from natural antioxidants are emerging as highly specific therapeutics for the treatment of various human diseases, including stroke, neurodegenerative pathologies, and cancer. However, the development of useful pseudo-natural nitrones requires the judicious choice of a secondary metabolite as precursor. Betalains are nitrogen-containing natural pigments that exhibit marked antioxidant and pharmacological properties and, hence, are ideal candidates for the design of multifunctional nitrones. In this work, we describe the semisynthesis and properties of a biocompatible and antioxidant betalain-nitrone named OxiBeet. This biobased compound is a better radical scavenger than ascorbic acid, gallic acid and most nonphenolic antioxidants and undergoes concerted proton-coupled electron transfer. The autoxidation of OxiBeet gives rise to a persistent nitroxide radical, which was studied by electron paramagnetic resonance spectroscopy. Femtosecond transient absorption spectroscopy reveals that excited state formation is not required for the oxidation of OxiBeet. The results are compared with those obtained using betanin, a natural betalain, and pBeet, the imine analogue of OxiBeet. The findings in this study will enable the development of antioxidant nitrones based on the novel $\mathrm{N}$-oxide 1,7-diazaheptamethinium scaffold and betalain dyes with enhanced hydrolytic stability in aqueous alkaline media

Keywords - nitrones, nitroxide, antioxidant, betalain, pseudo-natural compounds. 


\section{Introduction}

The formation of stable and persistent nitroxide adducts of free radical reactive species endows nitrones with marked antioxidant properties and support their use as therapeutic agents to treat disorders related to oxidative stress. ${ }^{1-3}$ The same process promotes the use of nitrones as building blocks for the construction of nitrogenous compounds ${ }^{4}$ and as electron paramagnetic resonance (EPR) spin traps. ${ }^{5}$ Although natural products containing the nitrone functional group are scarce, ${ }^{6}$ antioxidant and pharmacologically active compounds of natural origin can be used as starting materials for the semisynthesis of novel nitrones with high potential for application.

Antioxidants based on natural product scaffolds help to reduce the demand for nonrenewable hydrocarbon resources and are valuable in medicinal chemistry and drug discovery., 8 The nitrone derivatives of ligustrazine, a pyrazine found in the Chinese herb Ligusticum wallichii Franch., have antioxidant and thrombolytic properties and are effective therapeutics for treating ischemic stroke. ${ }^{3}$ Another example is the nitrone derived from the water-soluble analogue of vitamin E, Trolox, which shows enhanced radical scavenging and neuroprotective properties. ${ }^{9}$

Betalains are non-toxic water-soluble pigments found in plants, fungi and bacteria ${ }^{10}$ that have been consumed in foods for millennia. ${ }^{10-12}$ Indeed, betanin $(5-O-\beta$-glucosyl betanidin, EFSA/E162 and FDA/73.40) and indicaxanthin (L-proline-betaxanthin), the main pigments in red beetroots (Beta vulgaris L.) and prickly pears [Opuntia ficus-indica (L.) Mill.], respectively, have positive effects on the overall redox state in vivo. ${ }^{13-17}$ Both phenolic and non-phenolic betalains are highly efficient dietary antioxidants, ${ }^{14}$ outperforming vitamin $\mathrm{C}$, vitamin $\mathrm{E}$ and many flavonoids in terms of antioxidant capacity (thermodynamics, number of radicals scavenged) and activity (kinetics, reactivity towards radicals). ${ }^{14,18-20}$ 
Here, we describe the semisynthesis and properties of OxiBeet, the first betalain-nitrone. This bioinspired compound is stable towards hydrolysis under neutral and slightly alkaline conditions and is not cytotoxic to the human hepatic cell line HepaRG at concentration up to 1 $\mathrm{mM}$. OxiBeet has high radical scavenging capacity and low reduction potential. Plus, it can be converted into a persistent $\mathrm{N}$-oxide 1,7-diazaheptamethinium radical cation via autoxidation in aqueous solution. Comparison of the characteristics of OxiBeet with those of natural betalains and $\mathrm{pBeet}$ provide new insights into the use of polymethine dyes to develop resonance-stabilized radicals.

\section{Materials and methods}

\section{General information}

All chemicals were purchased from Sigma-Aldrich and used without further purification unless otherwise stated. Betalamic acid was extracted from base-hydrolyzed red beetroot juice and processed as described previously. ${ }^{21,}{ }^{22}$ Aqueous solutions were prepared using deionized water (18.2 $\mathrm{M} \Omega \mathrm{cm}$ at $25^{\circ} \mathrm{C}, \mathrm{TOC} \leq 4 \mathrm{ppb}$, Milli-Q, Millipore). Additional experimental details are available in the ESI.

\section{Semisynthesis of OxiBeet}

$N$-Phenylhydroxylamine (13.5 mg, $125 \mu \mathrm{mol}, 5.0$ equiv) was added to an aqueous solution of betalamic acid (5.3 mg, $25 \mu$ mol, 1.0 equiv) acidified with $\mathrm{HCl}(25 \mathrm{~mL}, \mathrm{pH} 3)$. The mixture was kept at $4 \pm 1{ }^{\circ} \mathrm{C}$ for $1 \mathrm{~h}$ and the resulting red-orange solution was subjected to flash gel permeation column chromatography (Sephadex LH-20, water, $1.5 \times 20 \mathrm{~cm}, 20 \mathrm{psi}$ ). The orangecolored fraction was lyophilized $\left(-80^{\circ} \mathrm{C}, 0.08\right.$ mbar) to obtain OxiBeet (phenylhydroxylaminebetaxanthin, $3.8 \mathrm{mg}, 50 \%$ ) as an orange solid. 
${ }^{1}$ H-NMR (800 MHz, $\left.\mathbf{D}_{\mathbf{2}} \mathbf{O}\right): \delta 8.32(\mathrm{~d}, J=10.4 \mathrm{~Hz}, 1 \mathrm{H}), 7.60(\mathrm{~d}, J=7.9 \mathrm{~Hz}, 2 \mathrm{H}), 7.52(\mathrm{t}, J=7.9$ Hz, 2H), 7.46-7.41 (m, 1H), 7.09 (d, $J=10.4 \mathrm{~Hz}, 1 \mathrm{H}), 6.50$ (bs, 1H), 4.27 (bs, 1H), 3.22 (bs, $1 \mathrm{H}), 3.10(\mathrm{dd}, J=17.1,7.5 \mathrm{~Hz}, 1 \mathrm{H})$.

${ }^{13}$ C-NMR (200 MHz, D 2 O): $\delta$ 179.84, 170.39, 160.41, 153.81, 146.46, 132.24, 131.53, 122.67, $118.30,109.77,57.02,30.51$.

HRMS (ESI(+)-TOF): Calc'd for $\mathrm{C}_{15} \mathrm{H}_{15} \mathrm{~N}_{2} \mathrm{O}_{5}{ }^{+}$, [M] ${ }^{+}, 303.0975$; found 303.0976; diff. 0.3 ppm.

UV-Vis: $\lambda_{\mathrm{abs}}{ }^{\max }=460 \mathrm{~nm}, \varepsilon^{460 \mathrm{~nm}}=35,000 \pm 2400 \mathrm{~L} \mathrm{~mol}^{-1} \mathrm{~cm}^{-1}$ (pH 6) (Figs. S1 and S2).

Fluorescence (FI): $\lambda_{\mathrm{Fl}}{ }^{\max }=562 \mathrm{~nm}\left(\mathrm{pH} 6, \lambda^{\mathrm{EX}}=460 \mathrm{~nm}\right)$.

\section{MTT assay}

HepaRG cells were cultured until confluence in Dulbecco's Modified Eagle Medium (DMEM) supplemented with $10 \%(\mathrm{v} / \mathrm{v})$ fetal bovine serum (FBS). Cells were seeded in a 96-well plate $\left(5 \times 10^{3}\right.$ cells $/$ well $)$ and treated with OxiBeet in $1 \%$ FBS DMEM $\left(\mathrm{mmol} \mathrm{L}^{-1}\right.$ to $\left.\mu \mathrm{mol} \mathrm{L} \mathrm{L}^{-1}\right)$ for $6 \mathrm{~h}$ at $37{ }^{\circ} \mathrm{C}$. Negative and positive control experiments were carried out using $1 \%$ FBS DMEM and Triton X-100 1\% (v/v) in phosphate buffer saline (PBS), respectively. A solution of MTT (3[4,5-dimethylthiazol-2-yl]-2,5-diphenyltetrazolium bromide) in 1\% FBS DMEM (5 mg mL ${ }^{-1}$, $10 \%(\mathrm{v} / \mathrm{v}))$ was added to each well. After incubating for $30 \mathrm{~min}$ at $37{ }^{\circ} \mathrm{C}$, the medium was removed and replaced with DMSO $(100 \mu \mathrm{L})$. The absorption of the resulting purple formazan solution was measured at $550 \mathrm{~nm}$, and the cell viability was calculated as the percentage of absorbance relative to the negative control. 


\section{Radical scavenging capacity}

Radical scavenging capacity was determined using the TEAC/ABTS ${ }^{+\cdot}$ assay. ${ }^{23}$ A solution of $\mathrm{ABTS}^{+\bullet} / \mathrm{ABTS}$ in phosphate buffer $\left(50 \mathrm{mmol} \mathrm{L}^{-1}, \mathrm{pH} 7.4\right)$ with an absorbance of 0.7 at $734 \mathrm{~nm}$ (46.7 $\mu \mathrm{mol} \mathrm{L}^{-1} \mathrm{ABTS}^{+*}$ ) was prepared. The bleaching of $\mathrm{ABTS}^{+\bullet}$ by the antioxidant $\left(\mu \mathrm{mol} \mathrm{L} \mathrm{L}^{-1}\right.$ concentration range) was monitored at $734 \mathrm{~nm}$ for $6 \mathrm{~min}$ at $25{ }^{\circ} \mathrm{C}$. The slope $(\alpha)$ of the linear correlation between the change in absorption $(\Delta \mathrm{Abs})$ and the antioxidant concentration was measured. The Trolox equivalent antioxidant capacity (TEAC) was calculated by dividing the value of $\alpha$ for the sample with that of the Trolox standard ( $\alpha_{\text {Sample }} / \alpha_{\text {Trolox }}$ ratio).

\section{Cyclic voltammetry}

Measurements were carried out at $25{ }^{\circ} \mathrm{C}$ on a Metrohm Autolab PGSTAT101 potentiostat/galvanostat equipped with a $10-\mathrm{mL}$ electrochemical cell and controlled using NOVA software. A glassy carbon working electrode (diameter of electrode disk $=2 \mathrm{~mm}$ ), a platinum wire auxiliary electrode, and an $\mathrm{Ag} / \mathrm{AgCl}(\mathrm{KCl}$ sat.) reference electrode were used; potential range: -0.2 to $0.8 \mathrm{~V}$, scan rate: $50 \mathrm{mV} \mathrm{s}^{-1}$; [analyte] $=88 \mathrm{mmol} \mathrm{L}^{-1}$ in aqueous $\mathrm{KCl}\left(0.1 \mathrm{~mol} \mathrm{~L}^{-1}\right)$ at $\mathrm{pH}$ 7.0. Before each experiment, the working electrode was polished with 0.05 - $\mu \mathrm{m}$-sized alumina particles ( 60 cycles) and washed with water under ultrasound irradiation for $1 \mathrm{~min}$.

\section{EPR spectroscopy and simulation}

X-band EPR spectra were obtained at room temperature $\left(20 \pm 2{ }^{\circ} \mathrm{C}\right)$ using a Bruker EMX spectrometer equipped with a standard cavity and operated at approximately $9.7 \mathrm{GHz}$ with a 100 $\mathrm{kHz}$ modulation frequency. The spectrometer settings were $20 \mathrm{~mW}$ microwave power, $0.5 \mathrm{G}$ modulation amplitude, $20.48 \mathrm{~ms}$ time constant, 1024 points, and $100 \mathrm{G}$ scan range. The 2,2-diphenyl-1-picrylhydrazyl $(\mathrm{DPPH})$ radical $(g$-value $=2.0036)$ was used to calibrate the magnetic field. pBeet and OxiBeet solutions $\left(1 \mathrm{mmol} \mathrm{L}^{-1}\right)$ were prepared immediately before use 
in aerated phosphate buffer $\left(50 \mathrm{mmol} \mathrm{L}^{-1}, \mathrm{pH}\right.$ 7.4) and kept at room temperature. All measurements were carried out using $200 \mu \mathrm{L}$ of the respective solution in a suprasil flat cell (Wilmad). EPR simulations were carried out using the Easyspin toolbox in MATLAB. ${ }^{24}$ The simulation was fitted to the experimental spectra using the Nelder-Mead simplex method. The following parameters were used: $g$-value $=2.0059 ; a_{\alpha}{ }^{\mathrm{N}}=33.6 \mathrm{MHz}, a_{\beta}{ }^{\mathrm{H}}=17.6 \mathrm{MHz}, a_{\gamma}{ }^{\mathrm{H}}=9.2$ $\mathrm{MHz}$ and $a_{\gamma}{ }^{2 \times \mathrm{H}}=8.2 \mathrm{MHz}$; line width for isotropic broadening and full width at half maximum $(\mathrm{FWHM})=0.01 \%$ Gaussian and $0.29 \%$ Lorentzian.

\section{Transient absorption spectroscopy}

Femtosecond pump-probe transient absorption measurements were performed using a regenerative amplified Ti:sapphire laser system (Libra Ultrafast Amplifier System designed by Coherent; $795 \mathrm{~nm}, \mathrm{FWHM} \approx 40 \mathrm{fs}, 1 \mathrm{~mJ} /$ pulse and $3 \mathrm{kHz}$ repetition rate) as the laser source and a Newport MS260i imaging spectrograph. Briefly, the $795 \mathrm{~nm}$ output pulse from the regenerative amplifier was split into two parts. The transmitted part was directed into an optical parametric amplifier (TOPAS-prime, Light Conversion) to generate a pump beam of $485 \mathrm{~nm}(1 \mathrm{~mW} \pm 2 \%)$, which was focused on the sample with a beam size of ca. $300-\mu \mathrm{m}$ in diameter. The reflected beam was passed through a delay stage ( $8.5 \mathrm{~ns} ; 1-2$ fs step size) and focused into a sapphire crystal to generate a white light continuum, which was used as the probe beam. The pump pulses were chopped by a synchronized chopper at $1500 \mathrm{~Hz}$ and the absorbance change was calculated with two adjacent probe pulses (pump-blocked and pump-unblocked). The samples were placed in 1-mm quartz cuvettes and measured under ambient conditions. 


\section{Results and discussion}

OxiBeet was semisynthesized as shown in Fig. 1a. Betalamic acid (1) was produced by the hydrolysis of the betalains in red beetroot juice, but it can also be obtained by enzymatic oxidation of L-DOPA ${ }^{25}$. The coupling between 1 and $N$-phenylhydroxylamine (2) in acidified water is quantitative and gives rise to OxiBeet (3). The product was isolated in $50 \%$ yield after purification in the presence of oxygen, which is twice as high as the values reported for other betalains. ${ }^{20,26}$ The high-resolution mass spectrum was consistent with the postulated structure of OxiBeet (Fig. 1b). Notably, both the semisynthesis and purification of $\mathbf{3}$ are carried out using water as solvent and $\mathbf{2}$ can be produced using environmentally benign methods. ${ }^{27}$

The high persistence and solubility of OxiBeet in water enables its characterization using nuclear magnetic resonance (NMR) spectroscopy (Fig. 1c), which is rarely used to study betalains because of their lability and low solubility in organic media. ${ }^{28,29}$ The coupling constant between the $\mathrm{H} 7$ and $\mathrm{H} 8$ atoms of OxiBeet $\left({ }^{3} J_{\mathrm{H} 7, \mathrm{H} 8}=10.4 \mathrm{~Hz}\right)$ is slightly smaller than the typical values typically reported for natural betalains $\left({ }^{3} \mathrm{~J}_{\mathrm{H} 7, \mathrm{H} 8} \sim 12 \mathrm{~Hz}\right){ }^{30}$ The $\mathrm{H} 7$ and $\mathrm{C} 5$ atoms of OxiBeet are deshielded (by ca. $1 \mathrm{ppm}$ and $10 \mathrm{ppm}$, respectively) compared to those of betanin and indicaxanthin ${ }^{28,30}$. Hence, the mesomeric donation of electron density by the oxygen atom of the nitrone group into the $\pi$-system decreases the electrophilicity of the $\mathrm{C} 8$ and increases the hydrolytic stability of OxiBeet compared to natural analogues. 


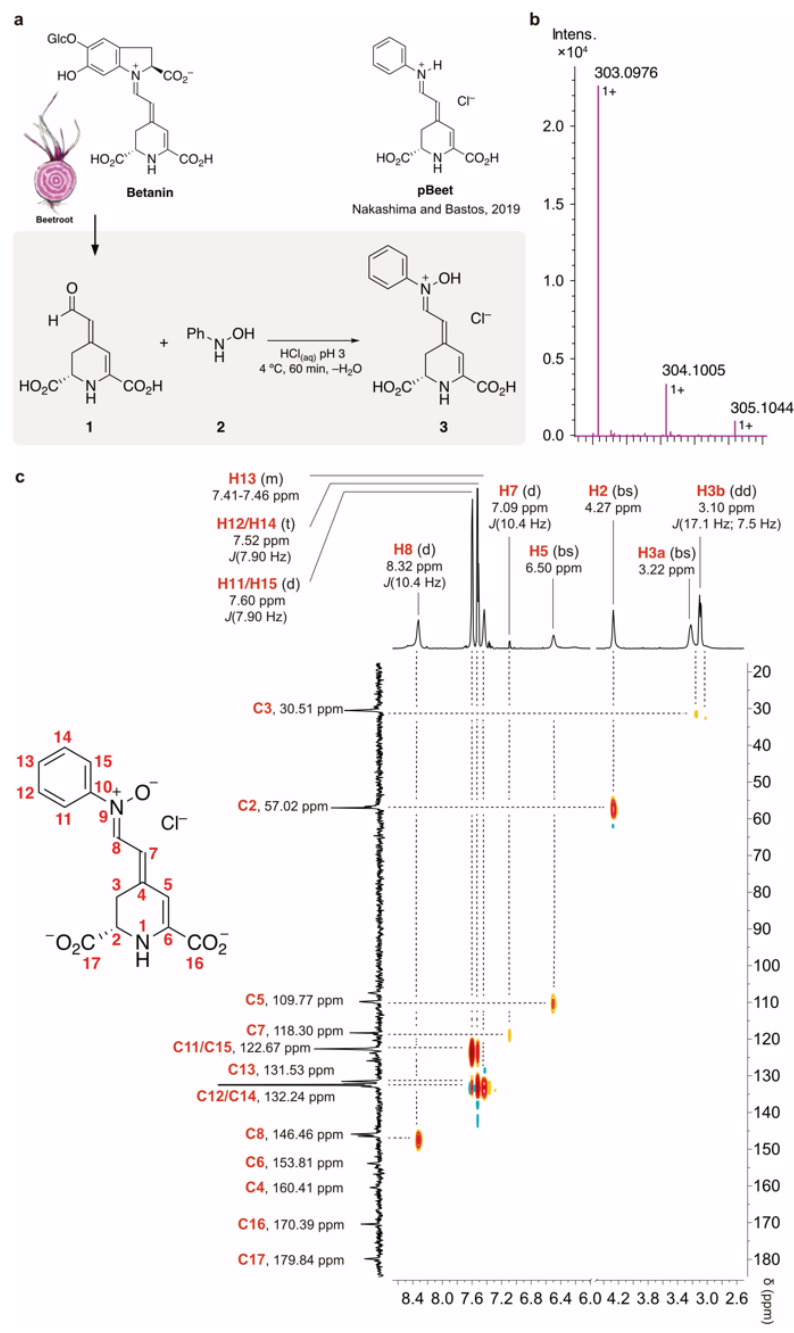

Figure 1. Semisynthesis and characterization of OxiBeet. (a) Acid-catalyzed coupling of betalamic acid (1) and $\mathbf{2}$ in water. The structures of betanin (main starting material of 1) and pBeet are shown for comparison. (b) High resolution mass spectrum of OxiBeet; $\mathrm{m} / \mathrm{z} 303.0976$ $[\mathrm{M}]^{+}$; Calc'd.: 303.0975. (c) ${ }^{1} \mathrm{H},{ }^{13} \mathrm{C}$, and $\left[{ }^{1} \mathrm{H},{ }^{13} \mathrm{C}\right]$ heteronuclear single quantum coherence (HSQC) spectra of OxiBeet (70 $\mu \mathrm{mol} \mathrm{L}^{-1}, 800 \mathrm{MHz} / 200 \mathrm{MHz}, \mathrm{D}_{2} \mathrm{O}$ at $288 \mathrm{~K}$ ). Atom numbering is shown in red; the assignment of the signals in the NMR spectra was supported by quantum mechanical GIAO calculations (Fig. S3). 
To examine the behavior of OxiBeet in aqueous solution, we compared the $\mathrm{pH}$ dependence of its observed first-order hydrolysis rate constant $\left(k_{\mathrm{obs}}\right)$ at $60{ }^{\circ} \mathrm{C}$ with those of pBeet and betanin (Figs. 2a and S4-S7). Under neutral and slightly alkaline conditions, OxiBeet was found to be far more stable than betanin. However, all compounds are subject to acid-catalyzed decomposition (Fig. 2a). Deprotonation of the nitrone group ( $\mathrm{p} K_{\mathrm{a}} 4.4$, Fig. S8) increases the negative charge density at the $\mathrm{N} 1$ of the 1,7-diazaheptamethinium system (Fig. 2b) and shifts the absorption maximum of OxiBeet to shorter wavelengths (blue shift, ca. $2000 \mathrm{~cm}^{-1}$, Fig. 2c). Such shift is not observed for pBeet, which ready undergoes hydrolysis in alkaline conditions. Natural betalains hydrolyze under alkaline conditions and the synthesis of a more stable analogue was reported to require extensive modification of the betalain framework. ${ }^{31}$

The biocompatibility of OxiBeet was tested against the human hepatic cell line HepaRG, which is a well-established model for studying drug metabolism and toxicity. ${ }^{32}$ Cells incubated with the betalain nitrone $\left(0.1 \mathrm{mmol} \mathrm{L}^{-1}\right.$ to $\left.1 \mu \mathrm{mol} \mathrm{L} \mathrm{L}^{-1}\right)$ for $6 \mathrm{~h}$ remained $100 \%$ viable, and 1 mmol L ${ }^{-1}$ OxiBeet was required to reduce cell viability by $8-10 \%$ (Fig. $2 \mathrm{~d}$ ). 


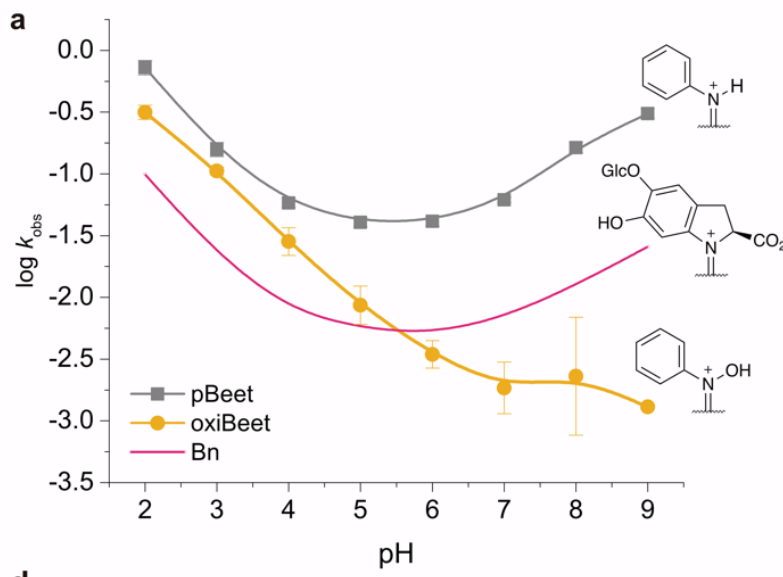

d
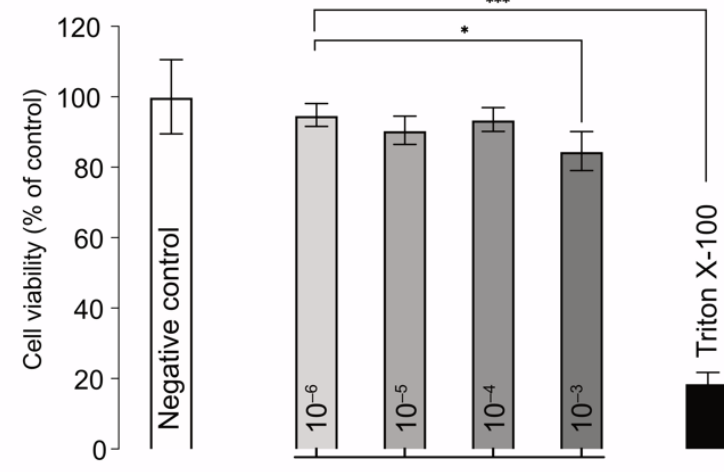
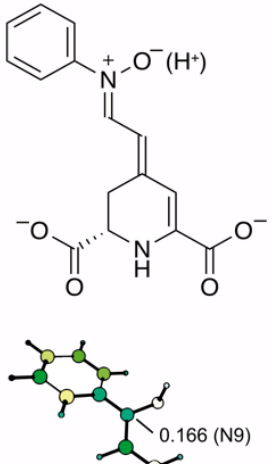

$\mu=35.3 \mathrm{D}$

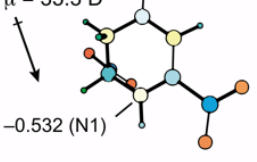

[oxiBeet] $\left(\mathrm{mol} \mathrm{L}^{-1}\right)$
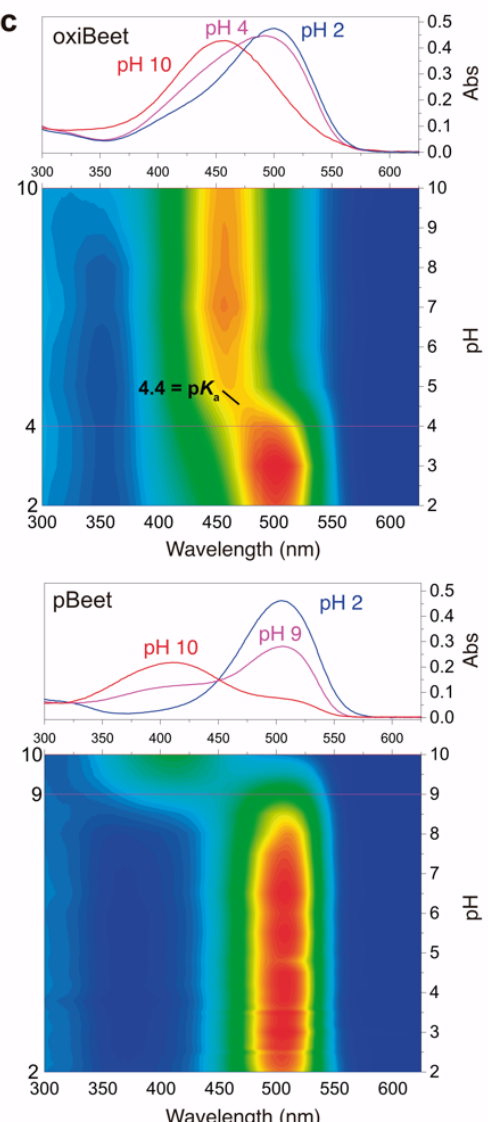

Figure 2. Properties of OxiBeet in aqueous solution. (a) Effect of the $\mathrm{pH}$ on the logarithm of observed rate constants $\left(k_{\mathrm{obs}}, \mathrm{min}^{-1}\right)$ for the decomposition of OxiBeet and pBeet at $60{ }^{\circ} \mathrm{C}$. Betanin is shown for reference; the data is reproduced from ref. ${ }^{33}$. (b) Partial charges of the nitrogen atoms of OxiBeet and its protonated $(\mathrm{N}-\mathrm{OH})$ form according to the Merz-KollmanSingh (MKS) scheme. (c) Contour plots of the absorbance spectra of OxiBeet and pBeet as a function of the $\mathrm{pH}$; red color indicates $\mathrm{Abs} \sim 0.5$. UV-Vis spectra at selected $\mathrm{pH}$ values are shown for clarity. (d) Viability of HepaRG cells treated with OxiBeet $\left(1 \mathrm{mmol} \mathrm{L}^{-1}\right.$ to $1 \mu \mathrm{mol} \mathrm{L}{ }^{-1}$, $6 \mathrm{~h}$ ) measured by using the MTT assay. Negative control experiments were carried out using 1\% FBS DMEM; cells incubated with Triton X-100 (1\% v/v in PBS) were used as positive controls. Asterisks indicate the value levels of statistical significance; $(*) P=0.02$ and $(* * *) P=0.0002$, 
one-way ANOVA with Geisser-Greenhouse correction and Dunnett's multiple comparison test, $N=5$.

The ability of nitrones to react with oxidants promotes their broad application. ${ }^{1,2}$ To assess the antioxidant potential of OxiBeet, we measured its radical scavenging capacity and redox potential. At $\mathrm{pH} 7.4$, the radical scavenging capacities of OxiBeet and pBeet determined using the $\mathrm{TEAC}_{\mathrm{ABTS}}{ }^{+\bullet}$ method $^{23}$ are identical at the $95 \%$ confidence level $(\mathrm{TEAC}=3.8 \pm 0.1$, Fig. 3a). However, the oxidation of the 1,7-diazaheptamethinium system of OxiBeet occurred at a much lower potential than those of other betalains, ${ }^{14}$ including $p B e e t,{ }^{34}$ as evidenced by the intense irreversible anodic response at $357 \mathrm{mV}$ vs. $\mathrm{Ag} \mid \mathrm{AgCl}$. Moreover, OxiBeet nitrone was reversibly oxidized to the corresponding nitroxide $\left(\mathrm{E}_{p \mathrm{a}}=166 \mathrm{mV}\right.$ and $\mathrm{E}_{p \mathrm{c}}=78 \mathrm{mV} ; \mathrm{E}_{1 / 2}=124$ $\mathrm{mV}$, Fig. 3b). Despite being non-phenolic, OxiBeet is a potent reducing agent and its TEAC is higher than those of well-known antioxidants such as gallic acid, vitamin $\mathrm{C}$, vitamin $\mathrm{E}$ and many flavonoids. ${ }^{35,36}$

The occurrence of concerted PCET, 37,38 that is, hydrogen atom transfer (HAT) or concerted proton-electron transfer (CPET), is the preferred thermodynamic pathway for the oxidation of OxiBeet (Fig. 3c). The homolytic bond dissociation energy (BDE) of the NO-H bond was at least $70 \mathrm{~kJ} \mathrm{~mol}^{-1}$ lower than the energy required for the electron transfer in sequential proton loss electron transfer (SPLET) or electron transfer followed by proton transfer $(\text { ET-PT) })^{39}$ (Fig. 3c). The change in enthalpy of the isodesmic reaction between the phenoxyl radical and OxiBeet was $-65 \mathrm{~kJ} \mathrm{~mol}^{-1}$, which was much lower than that of pBeet $\left(-11 \mathrm{~kJ} \mathrm{~mol}^{-}\right.$ 
$\left.{ }^{1}\right) \cdot{ }^{34}$ These results agree with the electrochemical and theoretical data and highlight the ease with which OxiBeet can be oxidized.

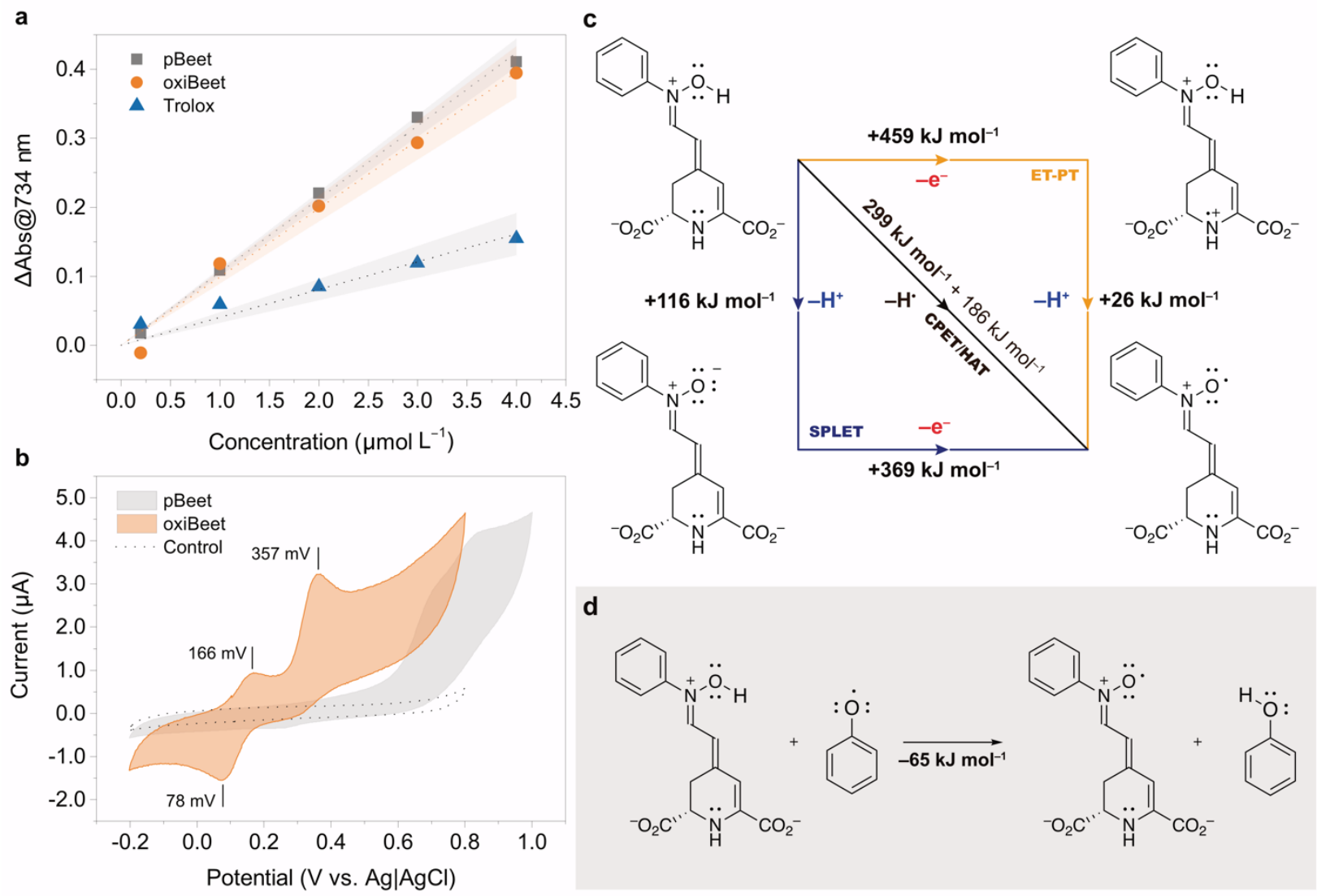

Figure 3. Antioxidant capacity and redox profile of OxiBeet. (a) Change in absorption ( $\Delta \mathrm{Abs})$ of $\mathrm{ABTS}^{+\bullet}$ at $734 \mathrm{~nm}$ after 6 min of reaction caused by different concentrations of OxiBeet, pBeet, and Trolox in phosphate buffer ( $\mathrm{pH} 7.4,50 \mathrm{mmol} \mathrm{L}^{-1}$ ). (b) Cyclic voltammograms of OxiBeet and pBeet in $0.1 \mathrm{~mol} \mathrm{~L}^{-1} \mathrm{KCl}$ and negative control (dotted line, $\left.0.1 \mathrm{~mol} \mathrm{~L}^{-1} \mathrm{KCl}_{(\mathrm{aq})}\right)$. Glassy carbon electrode, $\mathrm{Ag} \mid \mathrm{AgCl}\left(\mathrm{KCl}\right.$ sat.), scan rate: $50 \mathrm{mV} \mathrm{s}{ }^{-1}$, [betalain] $=88 \mathrm{mmol} \mathrm{L} \mathrm{L}^{-1}$. (c) More O'Ferrall-Jencks diagrams for the ionization and $1 \mathrm{e}^{-}-$ oxidation of OxiBeet. Compounds are presented as dicarboxylates since this is the expected 
major form in water at $\mathrm{pH}$ higher than $4 .{ }^{33}$ Energies refer to the enthalpy changes between states; the value of $186 \mathrm{~kJ} \mathrm{~mol}^{-1}$ in the concerted pathway is required for the $1 \mathrm{e}^{-}$-oxidation of $\mathrm{H}^{\bullet}$ to produce $\mathrm{H}^{+}$and $\mathrm{e}^{-}$. (d) Isodesmic reaction between the phenoxyl radical and OxiBeet to produce phenol and the OxiBeet ${ }^{+}$.

A solution of OxiBeet in phosphate buffer at $\mathrm{pH} 7.4$ was saturated with molecular oxygen for $1 \mathrm{~min}$ at room temperature to promote its autoxidation. The EPR spectrum of the product shows a 14 lines pattern, indicating a delocalized NO radical with the following hyperfine coupling constants (hfcc, in Gauss): $a_{\alpha}{ }^{\mathrm{N}}=11.9, a_{\beta}{ }^{\mathrm{H}}=6.3, a_{\gamma}{ }^{\mathrm{H}}=3.3$ and $a_{\gamma}{ }^{2 \mathrm{H}}=2.9$ (Fig. 4a). This result is compatible with the delocalized nitroxide 1,7-diazaheptamethinyl radical cation of OxiBeet, as supported by EPR simulation. Control experiments using pBeet did not show an EPR response. The value of $k_{\mathrm{obs}}$ for the formation of OxiBeet ${ }^{+\cdot}$ was $(6.5 \pm 1.4) \times 10^{-4} \mathrm{~s}^{-1}$ (halflife $\left(\tau / \frac{1}{2}\right)=18 \mathrm{~min}$, excess $\mathrm{O}_{2}$, pseudo-first order conditions), while its decomposition rate constant was much lower $\left(k_{\mathrm{obs}}=(8.8 \pm 2.7) \times 10^{-5} \mathrm{~s}^{-1}\right.$, lifetime $\left.(\tau)=2.5 \mathrm{~h}, \tau_{1 / 2}=106 \mathrm{~min}\right)($ Figs. $4 \mathrm{~b}$ and S9). For comparison, the $\tau \frac{1}{2}$ of the superoxide adducts of the widely used $N$-oxide spin traps DMPO, EMPO and CDMPO are 0.9, 9.9 and 27.5 min, respectively. ${ }^{40}$

The positive spin density of OxiBeet ${ }^{+*}$ is localized on the N1, C5, C7 and N9 atoms (Fig. $4 \mathrm{c}$ ), providing additional support to the assigned structure. The partial positive charges at the N1 and $\mathrm{N} 9$ atoms increase upon $1 \mathrm{e}^{-}$-oxidation of OxiBeet, as expected considering charge delocalization arguments. Although studies on the reaction between betanin and radicals were performed using EPR spectroscopy, ${ }^{41}$ this report shows for the first time that the 1,7-diazaheptamethinium scaffold increases the stability of nitroxide radicals. Last, these 
findings highlight the importance of the 1,7-diazaheptamethinium system of betalains for their striking antioxidant properties. ${ }^{20,34}$

Ultrafast transient absorption experiments were carried out to determine whether photoexcitation plays a role in the autoxidation of OxiBeet. In aerated water, the electronic excitation of OxiBeet at $485 \mathrm{~nm}$ results in ground state bleaching (Fig. 4d). The transient spectra revealed a positive absorption band at $450 \mathrm{~nm}$, which was ascribed to the excited state absorption $\left(\mathrm{S}_{1} \rightarrow \mathrm{S}_{n} ; n>1\right)$, and a negative band at about $520 \mathrm{~nm}$ that was attributed to $\mathrm{S}_{0}$ depletion. The broad negative absorption band centered at approximately $625 \mathrm{~nm}$ was attributed to the $\mathrm{S}_{1} \rightarrow \mathrm{S}_{0}$ stimulated emission, which was visible after $800 \mathrm{fs}$ and ceased to exist after $10 \mathrm{ps}$. In fact, full OxiBeet ground state recovery occurs in less than 100 ps after photoexcitation, indicating that $\mathrm{S}_{1} \rightarrow \mathrm{T}_{1}$ intersystem crossing is precluded and photoproducts were not formed in noticeable amounts, as observed for betanin ${ }^{42}$. 

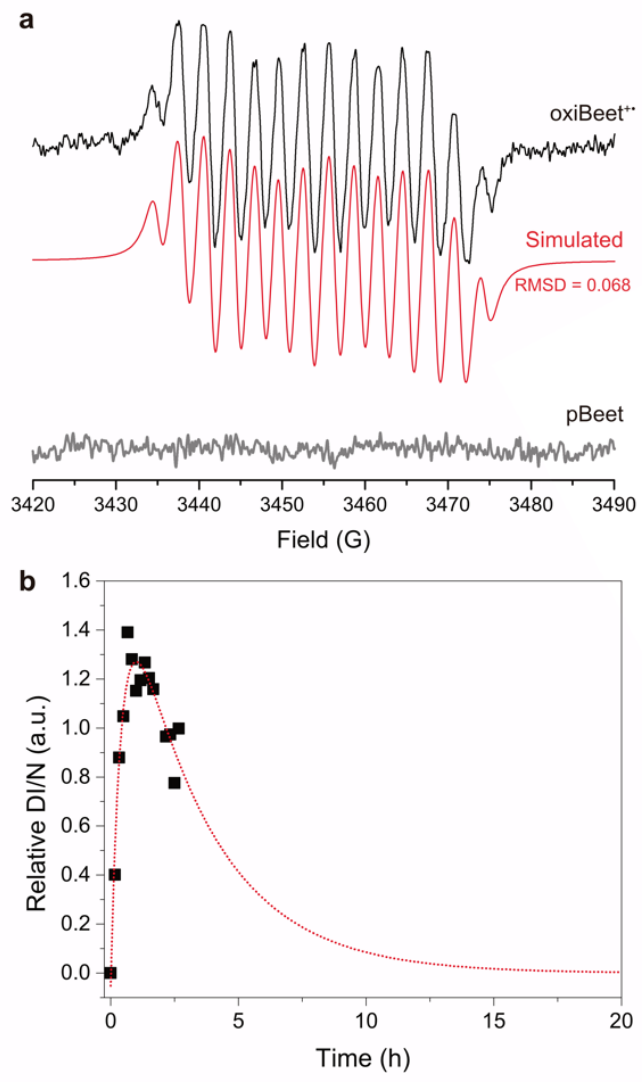

C
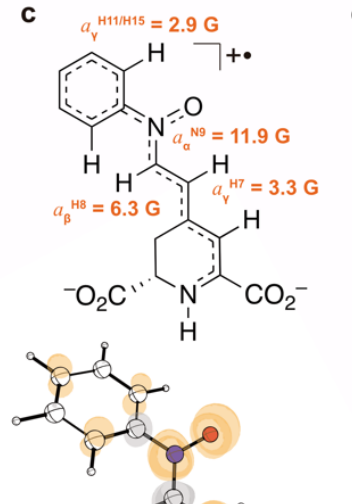

\footnotetext{
(1)

(1)
}

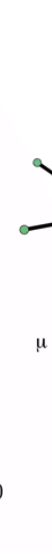

d
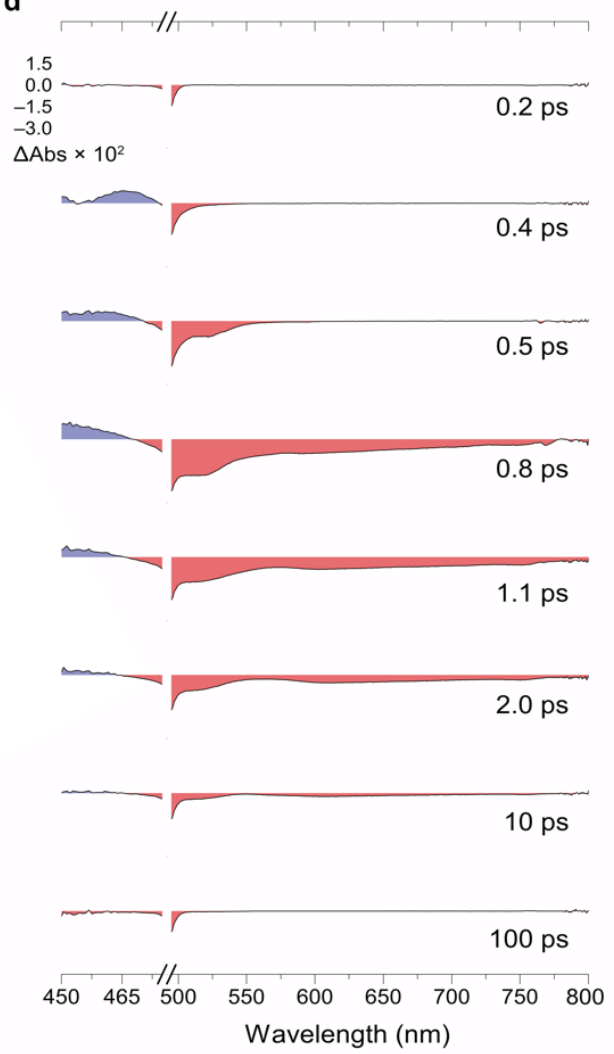

Figure 4. Electronic spin structure of OxiBeet ${ }^{+\bullet}$ and photophysical data. (a) Observed (black) and simulated (red) hyperfine EPR spectra of OxiBeet ${ }^{+\bullet}$ in phosphate buffer, $\mathrm{pH} 7.4$ at $298 \mathrm{~K}$. The lack of response of pBeet is shown for comparison. The color of the solution remained unchanged during the experiment. (b) Kinetics of formation and disappearance of OxiBeet ${ }^{+\bullet}$; the dotted red line represents nonlinear fitting of the data to a biexponential function. (c) Spin density distribution and partial charges of the nitrogen atoms of OxiBeet ${ }^{+*}$; red and blue indicate positive and negative spin densities, respectively. The values of the hfccs of OxiBeet ${ }^{+\bullet}$ calculated from the simulation are presented in orange. (d) Transient absorption spectra of OxiBeet in water; pump wavelength: $485 \mathrm{~nm}$. 


\section{Conclusions}

The repertoire of multifunctional nitrones has been expanded by the design and synthesis of a prototypical betalain-nitrone based on the $N$-oxide 1,7-diazaheptamethinium scaffold. OxiBeet is based on a chiral starting material obtained from renewable sources and can be conveniently synthesized and purified in water. This pseudo-natural compound is a potent antioxidant that is not cytotoxic within the concentration range typically used in biological assays. Moreover, it is oxidized via concerted PCTE and is stable towards hydrolysis under neutral and alkaline aqueous conditions, which is a clear advantage over its betalain counterparts. The formation of the resonance-stabilized cation radical of OxiBeet upon autoxidation enables the development of antioxidants that can inhibit oxygen-induced changes in industrial products and demonstrates the importance of the 1,7-diazaheptamethinium system for the antioxidant properties of betalains in general. These findings indicate that OxiBeet and its analogue betalain-nitrones can be potentially used as biocompatible antioxidants, redox mediators and spin traps, opening new perspectives for the development of therapeutics for diseases associated with oxidative stress.

\section{Author contribution}

E.L.B. conceived the study. E.L.B. and A.C.P wrote the paper. E.L.B., A.C.P., R.B.F., L.C.E., C.O.M., F.A.D., E.P., Y.H., J.S. and A.M.C.F. designed the experiments. A.C.P., R.B.F., L.C.E., C.O.M., F.A.D., and Y.H. performed the experimental work. E.L.B. carried out theoretical calculations. All authors discussed and interpreted the results.

\section{Conflicts of interest}

There are no conflicts to declare. 


\section{Acknowledgements}

We thank MSc. Janaina D. Vilcachagua (Analytical Central, IQUSP) for help with the NMR analyses, and Prof. Fabio Forti for providing us HepaRG cells. This work was partially supported by the São Paulo Research Foundation - FAPESP (ELB, 2014/22136-4, 2019/08950-1, 2019/06391-8 and 2019/15412-9; ACP 2015/18474-4; AMDCF 2013/07937-8), the Brazilian National Council for Scientific and Technological Development - CNPq (ELB, 301623/2019-8), and the Coordenação de Aperfeiçoamento de Pessoal de Nível Superior (CAPES, Finance Code 001).

\section{References}

1. Floyd, R. A.; Kopke, R. D.; Choi, C. H.; Foster, S. B.; Doblas, S.; Towner, R. A., Nitrones as therapeutics. Free Radic Biol Med 2008, 45 (10), 1361-74.

2. Oliveira, C.; Benfeito, S.; Fernandes, C.; Cagide, F.; Silva, T.; Borges, F., NO and HNO donors, nitrones, and nitroxides: Past, present, and future. Med Res Rev 2018, 38 (4), 11591187.

3. Marco-Contelles, J., Recent advances on nitrones design for stroke treatment. J Med Chem 2020, 63 (22), 13413-13427.

4. Murahashi, S. I.; Imada, Y., Synthesis and transformations of nitrones for organic synthesis. Chem Rev 2019, 119 (7), 4684-4716.

5. Bagryanskaya, E. G.; Krumkacheva, O. A.; Fedin, M. V.; Marque, S. R. A., Development and application of spin traps, spin probes, and spin labels. Method Enzymol 2015, 563, 365-396.

6. Waldman, A. J.; Ng, T. L.; Wang, P.; Balskus, E. P., Heteroatom-heteroatom bond formation in natural product biosynthesis. Chem Rev 2017, 117 (8), 5784-5863.

7. Rosselin, M.; Poeggeler, B.; Durand, G., Nitrone derivatives as therapeutics: From chemical modification to specific-targeting. Curr Top Med Chem 2017, 17 (18), 20062022.

8. Rodrigues, T.; Reker, D.; Schneider, P.; Schneider, G., Counting on natural products for drug design. Nat Chem 2016, 8 (6), 531-41.

9. Socrier, L.; Rosselin, M.; Gomez Giraldo, A. M.; Chantemargue, B.; Di Meo, F.; Trouillas, P.; Durand, G.; Morandat, S., Nitrone-Trolox conjugate as an inhibitor of lipid oxidation: Towards synergistic antioxidant effects. Biochim Biophys Acta, Biomembr 2019, 1861 (8), 1489-1501.

10. Quina, F. H.; Bastos, E. L., Chemistry inspired by the colors of fruits, flowers and wine. $A n$ Acad Bras Cienc 2018, 90 (1 Suppl 1), 681-695.

11. Prior, R. L.; Cao, G., Antioxidant phytochemicals in fruits and vegetables: diet and health implications. HortScience 2000, 35 (4), 588-592. 
12. Kugler, F.; Stintzing, F. C.; Carle, R., Evaluation of the antioxidant capacity of betalainic fruits and vegetables. J Appl Bot Food Qual 2007, 81 (1), 69-76.

13. Kanner, J.; Harel, S.; Granit, R., Betalains - A new class of dietary cationized antioxidants. J Agric Food Chem 2001, 49 (11), 5178-5185.

14. Slimen, I. B.; Najar, T.; Abderrabba, M., Chemical and antioxidant properties of betalains. J Agric Food Chem 2017, 65 (4), 675-689.

15. Ciriminna, R.; Fidalgo, A.; Danzì, C.; Timpanaro, G.; Ilharco, L. M.; Pagliaro, M., Betanin: A bioeconomy insight into a valued betacyanin. ACS Sust Chem Eng 2018, 6 (3), 2860-2865.

16. Rahimi, P.; Abedimanesh, S.; Mesbah-Namin, S. A.; Ostadrahimi, A., Betalains, the nature-inspired pigments, in health and diseases. Crit Rev Food Sci 2019, 59 (18), 29492978.

17. Hadipour, E.; Taleghani, A.; Tayarani-Najaran, N.; Tayarani-Najaran, Z., Biological effects of red beetroot and betalains: a review. Phytother Res 2020, 34 (8), 1847-1867.

18. Apak, R.; Ozyurek, M.; Guclu, K.; Capanoglu, E., Antioxidant activity/capacity measurement. 1. Classification, physicochemical principles, mechanisms, and electron transfer (ET)-based assays. J Agric Food Chem 2016, 64 (5), 997-1027.

19. Polturak, G.; Aharoni, A., "La vie en rose": Biosynthesis, sources, and applications of betalain pigments. Mol Plant 2018, 11 (1), 7-22.

20. Gonçalves, L. C. P.; Lopes, N. B.; Augusto, F. A.; Pioli, R. M.; Machado, C. O.; Freitas-Dörr, B. C.; Suffredini, H. B.; Bastos, E. L., Phenolic betalain as antioxidants: meta means more. Pure Appl Chem 2020, 92 (2), 243-253.

21. Pioli, R. M.; Mattioli, R. R.; Esteves, L. C.; Dochev, S.; Bastos, E. L., Comparison of the effect of $N$-methyl and $N$-aryl groups on the hydrolytic stability and electronic properties of betalain dyes. Dyes Pigm 2020, 183, 108609.

22. Schliemann, W.; Kobayashi, N.; Strack, D., The decisive step in betaxanthin biosynthesis is a spontaneous reaction. Plant Physiol 1999, 119 (4), 1217-1232.

23. Re, R.; Pellegrini, N.; Proteggente, A.; Pannala, A.; Yang, M.; Rice-Evans, C., Antioxidant activity applying an improved ABTS radical cation decolorization assay. Free Radic Biol Med 1999, 26 (9-10), 1231-1237.

24. Stoll, S.; Schweiger, A., EasySpin, a comprehensive software package for spectral simulation and analysis in EPR. J Magn Reson 2006, 178 (1), 42-55.

25. Polturak, G.; Aharoni, A., Advances and future directions in betalain metabolic engineering. New Phytol 2019, 224 (4), 1472-1478.

26. Rodrigues, A. C. B.; Mariz, I. D. A.; Macoas, E. M. S.; Tonelli, R. R.; Martinho, J. M. G.; Quina, F. H.; Bastos, E. L., Bioinspired water-soluble two-photon fluorophores. Dyes Pigm 2018, 150, 105-111.

27. Doherty, S.; Knight, J. G.; Backhouse, T.; Summers, R. J.; Abood, E.; Simpson, W.; Paget, W.; Bourne, R. A.; Chamberlain, T. W.; Stones, R.; Lovelock, K. R. J.; Seymour, J. M.; Isaacs, M. A.; Hardacre, C.; Daly, H.; Rees, N. H., Highly selective and solventdependent reduction of nitrobenzene to n-phenylhydroxylamine, azoxybenzene, and aniline catalyzed by phosphino-modified polymer immobilized ionic liquid-stabilized AuNPs. ACS Catal 2019, 9 (6), 4777-4791.

28. Stintzing, F. C.; Kugler, F.; Carle, R.; Conrad, J., First C-13-NMR assignments of betaxanthins. Helv Chim Acta 2006, 89 (5), 1008-1016. 
29. Wybraniec, S.; Nowak-Wydra, B.; Mizrahi, Y., H-1 and C-13 NMR spectroscopic structural elucidation of new decarboxylated betacyanins. Tetrah Lett 2006, 47 (11), 1725 1728.

30. Stintzing, F. C.; Conrad, J.; Klaiber, I.; Beifuss, U.; Carle, R., Structural investigations on betacyanin pigments by LC NMR and 2D NMR spectroscopy. Phytochemistry 2004, 65 (4), 415-422.

31. Freitas-Dörr, B. C.; Machado, C. O.; Pinheiro, A. C.; Fernandes, A. B.; Dörr, F. A.; Pinto, E.; Lopes-Ferreira, M.; Abdellah, M.; Sa, J.; Russo, L. C.; Forti, F. L.; Gonçalves, L. C. P.; Bastos, E. L., A metal-free blue chromophore derived from plant pigments. Sci Adv 2020, 6 (15), eaaz0421.

32. Donato, M. T.; Jover, R.; Gomez-Lechon, M. J., Hepatic cell lines for drug hepatotoxicity testing: Limitations and strategies to upgrade their metabolic competence by gene engineering. Curr Drug Metab 2013, 14 (9), 946-968.

33. Esteves, L. C.; Pinheiro, A. C.; Pioli, R. M.; Penna, T. C.; Baader, W. J.; Correra, T. C.; Bastos, E. L., Revisiting the mechanism of hydrolysis of betanin. Photochem Photobiol 2018, 94 (5), 853-864.

34. Nakashima, K. K.; Bastos, E. L., Rationale on the high radical scavenging capacity of betalains. Antioxidants 2019, 8 (7), 222.

35. Foti, M. C.; Amorati, R., Non-phenolic radical-trapping antioxidants. J Pharmacy Pharmacol 2009, 61 (11), 1435-1448.

36. Gulcin, I., Antioxidants and antioxidant methods: an updated overview. Arch Toxicol 2020, 94 (3), 651-715.

37. Darcy, J. W.; Koronkiewicz, B.; Parada, G. A.; Mayer, J. M., A continuum of protoncoupled electron transfer reactivity. Acc Chem Res 2018, 51 (10), 2391-2399.

38. Warren, J. J.; Tronic, T. A.; Mayer, J. M., Thermochemistry of proton-coupled electron transfer reagents and its implications. Chem Rev 2010, 110 (12), 6961-7001.

39. Weinberg, D. R.; Gagliardi, C. J.; Hull, J. F.; Murphy, C. F.; Kent, C. A.; Westlake, B. C.; Paul, A.; Ess, D. H.; McCafferty, D. G.; Meyer, T. J., Proton-coupled electron transfer. Chem Rev 2012, 112 (7), 4016-93.

40. Ouari, O.; Hardy, M.; Karoui, H.; Tordo, P., Recent developments and applications of the coupled EPR/Spin trapping technique (EPR/ST). In Electron Paramagnetic Resonance, Gilbert, B. C.; Murphy, D. M.; Chechik, V., Eds. Royal Society of Chemistry: Cambridge, 2010; Vol. 22, pp 1-40.

41. Esatbeyoglu, T.; Wagner, A. E.; Motafakkerazad, R.; Nakajima, Y.; Matsugo, S.; Rimbach, G., Free radical scavenging and antioxidant activity of betanin: electron spin resonance spectroscopy studies and studies in cultured cells. Food Chem Toxicol 2014, 73, 119-26.

42. Wendel, M.; Nizinski, S.; Tuwalska, D.; Starzak, K.; Szot, D.; Prukala, D.; Sikorski, M.; Wybraniec, S.; Burdzinski, G., Time-resolved spectroscopy of the singlet excited state of betanin in aqueous and alcoholic solutions. Phys Chem Chem Phys 2015, 17 (27), 18152-8. 